\title{
On inverse problem of Choquet integral
}

\section{Zhao Zhang and Zeshui Xu*}

College of Sciences, PLA University of Science and Technology, Nanjing, Jiangsu 210007, China

\begin{abstract}
The Choquet integral is a popular tool for dealing with multiple criteria decision making. In practice, if we have a fuzzy measure on the set of criteria, we can use Choquet integral to rank alternatives. In this paper, we consider the inverse problem of Choquet integral: Given a ranking of alternatives, does there exist a fuzzy measure by which we can get the ranking through Choquet integral.
\end{abstract}

Keywords: Choquet integral, inverse problem, multiple criteria decision making, fuzzy measure, ranking

\section{Introduction}

The Choquet integral [1] is a generalization of the Lebesgue integral, and like it, defined with respect to a measure. Instead of Lebesgue measure, Choquet measure is defined with respect to fuzzy measures. Fuzzy measures have been a very useful tool in multiple criteria decision making, since introduced in 1974 by Sugeno [2]. The theory of fuzzy measures has become a huge subject [3-5]. Fuzzy measures can model uncertainty and interactions among criteria. At the beginning of nineties, Sugeno integral [2] was the main tool to compute an average global score with a fuzzy measure expressing the importance of criteria. Then, Murofushi and Sugeno [6,7] proposed to use the Choquet integral. It quickly became popular and has been widely used in many decision making situations. One reason for its popularity is that Choquet integral is a generalization of the weighted arithmetic mean and the ordered weighted average (OWA) [8]. If we have a fuzzy measure on the set of criteria, it's easy to rank alternatives through Choquet integral.

In this paper, we discuss the inverse problem of Choquet integral: Given a ranking of alternatives, does there exist a fuzzy measure by which we can get the ranking through Choquet integral? The inverse problem of Choquet integral, first of all, is theoretically important. In some multiple criteria decision making situations, it's difficult to define a fuzzy measure on the set of criteria and rank alternatives with Choquet integral. Then, it should be quite useful, if we can easily decide whether a ranking of alternatives is realizable through Choquet integral or not. Our main aim is not to get a fuzzy measure, but to test the existence of some fuzzy measures.

\section{Fuzzy measures and Choquet integral}

Let's start with some definitions:

Definition 1: (Sugeno [2]). Let $\mathcal{N}=\{1,2, \ldots, n\}$, a fuzzy measure is a set function $v(A) \leq v(B)$ which is monotonic (i.e. $v(A) \leq v(B)$ whenever $A \subset B \subset \mathcal{N}$ ) and satisfies $v(\varnothing)=0$ and $v(\mathcal{N})=1$.

To define a fuzzy measure on $\mathcal{N}$, we need to define a real number for all the $2^{n}-2$ nontrivial subsets of $\mathcal{N}$, which is a difficult task in many applications of fuzzy measure. In many applications, some particular measures have been used, like decomposable measures [9], $\lambda$-measures $[2,10]$, possibility measures $[11,12], k$-additive measure $[13,14]$, etc.

Definition 2: (Choquet [1]). The discrete Choquet integral with respect to a fuzzy measure $v$ is given by

$$
C_{v}(\mathbf{x})=\sum_{i=1}^{n}\left(x_{(i)}-x_{(i-1)}\right) v\left(H_{i}\right)
$$

where $\mathbf{x}=\left(x_{(1)}, x_{(2)}, \ldots, x_{(n)}\right)$ is the non-decreasing permutation of a real vector $\mathbf{x}=\left(x_{1}, x_{2}, \ldots, x_{n}\right)$ and we assume $x_{(1)} \geq x_{(0)}=0$, and $H_{i}=\{(i), \ldots,(n)\}$ is the subset of indexes of the $n-i+1$ largest components of $\mathbf{x}$.

In a multiple criteria decision making problem, we have alternatives $m$ and $n$ criteria. If we have a fuzzy measure on the set of criteria, then we can use Choquet integral to rank alternatives. Here we give a simple example: Let's consider two criteria $B_{1}$ and $B_{2}$, and three alternatives $A_{1}, A_{3}$, and $A_{3}$. The evaluations of the alternatives $A_{i}(i=1,2,3)$ on each criterion $B_{j}$ is given below:

\begin{tabular}{|c|c|c|}
\hline & $B_{1}$ & $B_{2}$ \\
\hline$A_{1}$ & 0.3 & 0.4 \\
\hline$A_{2}$ & 0.5 & 0.2 \\
\hline$A_{3}$ & 0.3 & 0.3 \\
\hline
\end{tabular}

If we define the fuzzy measure $v$ as: $v\left(\left\{B_{1}\right\}\right)=0.3$, $v\left(\left\{B_{2}\right\}\right)=0.5, v\left(\left\{B_{1}, B_{2}\right\}\right)=1$, then we have Choquet integrals for each alternative $A_{i}$ :

$$
\begin{aligned}
& C_{v}\left(A_{1}\right)=0.3 v\left(\left\{B_{1}, B_{2}\right\}\right)+(0.4-0.3) v\left(\left\{B_{2}\right\}\right)=0.35 \\
& C_{v}\left(A_{2}\right)=0.2 v\left(\left\{B_{1}, B_{2}\right\}\right)+(0.5-0.2) v\left(\left\{B_{1}\right\}\right)=0.29 \\
& C_{v}\left(A_{3}\right)=0.3 v\left(\left\{B_{1}, B_{2}\right\}\right)+(0.3-0.3) v\left(\left\{B_{2}\right\}\right)=0.3
\end{aligned}
$$

So we should rank the three alternatives as $A_{1} \succ A_{3} \succ A_{2}$, where " $\succ$ " means "be superior to".

\section{Inverse problem}

In the example of the last section, a fuzzy measure has been already defined, we can use Choquet integral to rank the alternatives. Sometimes, we need to know whether there exists a fuzzy measure, by which we can get a particular ranking of alternatives through Choquet integral. For instance, does there exist a fuzzy measure $v^{\prime}$ such that $C_{v^{\prime}}\left(A_{1}\right) \geq C_{v^{\prime}}\left(A_{3}\right)$

${ }^{*}$ Corresponding author: Zeshui Xu, College of Sciences, PLA University of Science and Technology, Nanjing, Jiangsu 210007, China, Tel: +86 25 84483382; E-mail: xuzeshui@263.net

Received February 17, 2014; Accepted March 10, 2014; Published March 17, 2014

Citation: Zhang Z, Xu ZS (2014) On inverse problem of Choquet integral. Ind Eng Manage 3: 125. doi: 10.4172/2169-0316.1000125

Copyright: ( 2014 Zhang Z, et al. This is an open-access article distributed under the terms of the Creative Commons Attribution License, which permits unrestricted use, distribution, and reproduction in any medium, provided the original author and source are credited. 
We call this kind of problem the inverse problem. Because defining a general fuzzy measure is of exponential complexity, the inverse problems seem to be quite difficult. However, the number of variables actually involving in computation is much smaller, and we can solve the inverse problems effectively. We will investigate the method in the rest part of the section.

We assume that there are $m$ alternatives and $n$ criteria. The evaluation of the alternative $A_{i}$ on the criterion $B_{j}$ is denoted as $x_{j}^{i} . \quad \mathbf{x}^{i}=\left(x_{(1)}^{i}, x_{(2)}^{i}, \ldots, x_{(n)}^{i}\right)$ It is the non-decreasing permutation of $\mathbf{x}^{i}=\left(x_{1}^{i}, x_{2}^{i}, \ldots, x_{n}^{i}\right) . \quad H_{l}^{i}=\{(l), \ldots,(n)\}$ is the subset of indexes of the $n-l+1$ largest components of $\mathbf{x}^{i} . r=(1,2,3 \ldots, m)$ is a permutation of $(1,2,3 \ldots, m)$. We need to find a fuzzy measure $v$, such that: $C_{v}\left(\mathbf{x}^{r_{i}}\right) \geq C_{v}\left(\mathbf{x}^{r_{i-1}}\right), i=2, \ldots, m$

In Eq.(3.1), the variables are $v\left(H_{l}^{i}\right)(i=1,2, \ldots, m, l=2, \ldots, n)$. The number of variables is less than $m(n-1)$, and may be identical for some $H_{l}^{i}$.

Since $v$ is a fuzzy measure, we have

$$
\left\{\begin{array}{c}
0 \leq v\left(H_{l}^{i}\right) \leq 1 \\
v\left(H_{l}^{i}\right) \leq v\left(H_{l^{\prime}}^{i^{\prime}}\right), \text { if } H_{l}^{i} \subseteq H_{l^{\prime}}^{i^{\prime}}
\end{array}\right.
$$

in which the number of inequalities is less than $2 m(n-1)+\left(\begin{array}{c}m(n-1) \\ 2\end{array}\right)$.

The inverse problem is equal to the feasibility problem of the inequality system:

$$
\left\{\begin{array}{c}
C_{v}\left(\mathbf{x}^{r_{i}}\right) \geq C_{v}\left(\mathbf{x}^{i_{i-1}}\right) \\
v\left(H_{l}^{i}\right) \leq v\left(H_{l^{\prime}}^{i^{\prime}}\right), \text { if } H_{l}^{i} \subseteq H_{l^{\prime}}^{i^{\prime}} \\
0 \leq v\left(H_{l}^{i}\right) \leq 1
\end{array}\right.
$$

which has at most $m(n-1)$ variables and $m-1+\left(\begin{array}{c}m n \\ 2\end{array}\right)+m(n-1)$ inequalities.

Mathematically, finding a solution of linear inequality system equals solving a linear programming problem. So finding a solution of Eq.(3.3) equals solving the LP problem below:

$$
\text { s.t. }\left\{\begin{array}{c}
C_{v}\left(\mathbf{x}^{r_{i}}\right) \geq C_{v}\left(\mathbf{x}^{r_{i-1}}\right) \\
v\left(H_{l}^{i}\right) \leq v\left(H_{l^{\prime}}^{i^{\prime}}\right), \text { if } H_{l}^{i} \subseteq H_{l^{\prime}}^{i^{\prime}} \\
0 \leq v\left(H_{l}^{i}\right) \leq 1
\end{array}\right.
$$

According to the discussion above, the number of variables and inequalities of the LP problem (3.4) is polynomials of $n$ and $m$ . Widely-used methods like the simplex method and the inner point method can solve this kind of problem quite effectively. By those methods, we can know whether there exists a feasible solution and find a feasible solution (if exists). However, a feasible solution of the LP problem does not define a complete fuzzy measure but only defines a part of a fuzzy measure.

Here, optimization is just a tool to solve inequality system problems. There are other ways to solve the feasibility problem of inequality system problems.

Here we still use the example of last section. To find a fuzzy measure $v^{\prime}$, such that $C_{v^{\prime}}\left(A_{2}\right) \geq C_{v^{\prime}}\left(A_{1}\right) \geq C_{v^{\prime}}\left(A_{3}\right)$. We can easily have the LP problem for the inverse problem:

$$
\text { s.t. }\left\{\begin{array}{c}
0.2+0.3 v^{\prime}\left(\left\{B_{1}\right\}\right) \geq 0.3+0.1 v^{\prime}\left(\left\{B_{2}\right\}\right) \\
0.3+0.1 v^{\prime}\left(\left\{B_{2}\right\}\right) \geq 0.3 \\
0 \leq v^{\prime}\left(\left\{B_{1}\right\}\right), v^{\prime}\left(\left\{B_{2}\right\}\right) \leq 1
\end{array}\right.
$$

Solving this LP problem, we can use Matlab command "linprog". Then, we get $v^{\prime}\left(\left\{B_{1}\right\}\right)=v^{\prime}\left\{\left(B_{2}\right)\right\}=1$, which is a feasible solution. In fact,

$$
\begin{aligned}
& C_{v^{\prime}}\left(A_{1}\right)=0.3 v^{\prime}\left(\left\{B_{1}, B_{2}\right\}\right)+(0.4-0.3) v^{\prime}\left(\left\{B_{2}\right\}\right)=0.4 \\
& C_{v^{\prime}}\left(A_{2}\right)=0.2 v^{\prime}\left(\left\{B_{1}, B_{2}\right\}\right)+(0.5-0.2) v^{\prime}\left(\left\{B_{1}\right\}\right)=0.5 \\
& C_{v^{\prime}}\left(A_{3}\right)=0.3 v^{\prime}\left(\left\{B_{1}, B_{2}\right\}\right)+(0.3-0.3) v^{\prime}\left(\left\{B_{2}\right\}\right)=0.3
\end{aligned}
$$

So we have $C_{v^{\prime}}\left(A_{2}\right) \geq C_{v^{\prime}}\left(A_{1}\right) \geq C_{v^{\prime}}\left(A_{3}\right)$.

In practical situations, if we use some particular measures, like decomposable measures [9], $\lambda$-measures $[2,10]$, possibility measures $[11,12], k$-additive measure $[13,14]$, etc. the number of variables and inequalities will be further reduced, which makes inverse problem easier.

\section{Conclusions}

In this paper, we have discussed the inverse problem of Choquet integral in multiple criteria decision making and proposed a LP programming method to solve it. We have shown that solving the inverse problems is of polynomial complexity, and the proposed methods can solve it effectively.

\section{Acknowledgment}

The work was supported in part by the National Natural Science Foundation of China (No. 61273209).

\section{References}

1. Choquet G (1953) Theory of capacities, Annales de l'institut Fourier 5: 131-295

2. Sugeno M (1974) Theory of fuzzy integrals and its applications, Tokyo Institute of Technology, Tokyo

3. Denneberg D (1994) Non-additive measure and integral. Dordrecht: Kluwer Academic Publishers.

4. Pap E (2002) Handbook of measure Theory: In two volumes, Amsterdam Elsevier.

5. Wang Z, Klir GJ (1992) Fuzzy measure theory. New York: Springer.

6. Murofushi T (1993) Some quantities represented by the choquet integral. Fuzzy Sets and Systems 56: 229-235.

7. Sugeno M, Murofushi $T$ (1987) Choquet integral as an integral form for a general class of fuzzy measures, in: 2nd IFSA Congress: 408-411.

8. Yager RR (1988) On ordered weighted averaging aggregation operators in multicriteria decision making, IEEE, Systems, Man and Cybernetics 18: 183190

9. Weber $S$ (1984) Decomposable measures and integrals for archimedean t-conorms. J Math. Anal Appl 101: 114-138.

10. Sugeno M (1977) Fuzzy measures and fuzzy integrals: A survey, in Fuzzy Automata and Decision Processes 78: 89-102.

11. Zadeh LA (1999) Fuzzy sets as a basis for a theory of possibility. Fuzzy Sets and Systems 100: 9-34.

12. Dubois D, Prade H (1988) Possibility theory. Boston: Springer.

13. Grabisch M (1996) K-order additive fuzzy measures, in: In 6th Int. Conf. on In-formation Processing and Management of Uncertainty in Knowledge-Based Systems (IPMU): 1345-1350

14. Grabisch M (1997) K-order additive discrete fuzzy measures and thei representation, Fuzzy Sets and Systems 92: 167-189. 